\title{
A Natural Morphing Between Two Epicycloids
}

\author{
Francisco Javier González Vieli \\ (Communicated by Levent Kula)
}

\begin{abstract}
Let $A$ be a point which moves with constant angular speed on the circle with centre $O$ and radius 1, and $B$ a point wich moves with an angular speed which is $q$ times $(q>1)$ that of $A$ on the circle with centre $O$ and radius $r(r>1)$. We study the envelope of the straight lines $A B$. Both limit cases $r=1$ and $r=+\infty$ (with $q$ constant) are epicycloids; the relation between the shapes of these epicycloids does not depend on $q$.
\end{abstract}

Keywords: envelope; straight line; angular speed; epicycloid.

AMS Subject Classification (2010): Primary: 53A04 ; Secondary: 58K05.

\section{Introduction.}

An epicycloid is the trajectory of a point on a circle which rolls without slipping around another circle; the ratio of the radii of these circles determines the shape of the epicycloid. Examples of epicycloids are the cardioid and the nephroid. My attention was drawn to epicycloids by a recent article [4] where it is shown by direct calculations how the cardioid can be obtained as the envelope of the family of chords joining a point to its 'double' on a circle. Now, this result is not new: it is exercise 5.7(2) in [1, p.185], where a generalization is hinted at. In fact, the general case is already in Gomes Teixeira's three volume work [2, volume II, §561 p.166]. It can be worded as follows.

On the circle $\alpha$ with centre $O:=(0,0)$ and radius 1 , let $A$ and $B$ be two points which, starting from $(1,0)$, move with constant speed. If the angular velocity of $B$ is $q$ times $(q>1)$ that of $A$, the chord $A B$ generates a family of straight lines whose envelope is an epicycloid (see Figure 1 for the case $q=4$ ).

Here we investigate what occurs if $B$ moves on the circle $\beta$ with centre $O$ and radius $r(r>1)$ starting from $(r, 0)$, its angular velocity being always $q$ times that of $A$. We calculate explicitly the parametric representation of the curve which is the envelope of the straight lines $A B$; we find the critical points of this curve and we study its position relatively to $\alpha$. The curve is not an epicycloid, but, as $r$ tends to 1 , we evidently get the epicycloid from Gomes Teixeira's cited result; moreover, as $r$ tends to $+\infty$, we also get an epicycloid. We show that the shapes of these epicycloids are related in a way which does not depend on $q$.

\section{Envelope and curve.}

We may suppose that $A$ and $B$ start at $t=0$ from the points $(1,0)$ and $(r, 0)$ respectively; we can then choose the following parametrizations:

$$
A(t)=(\cos t, \sin t), \quad B(t)=(r \cos q t, r \sin q t) .
$$

The straight line through $A$ and $B$ is given by the equation

$$
\frac{x-\cos t}{r \cos q t-\cos t}=\frac{y-\sin t}{r \sin q t-\sin t} .
$$




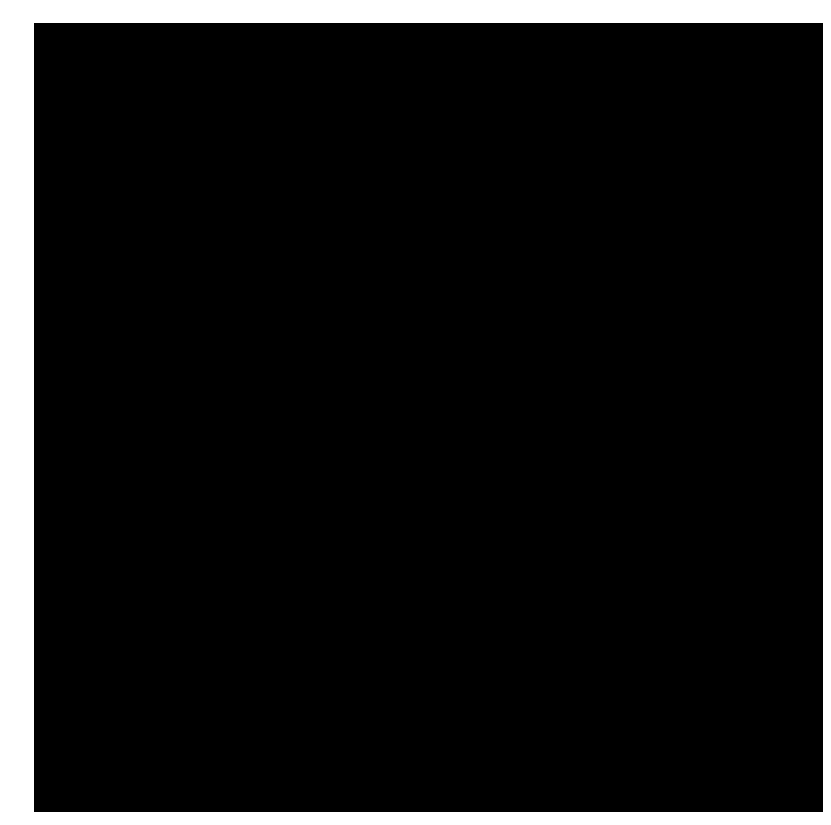

Figure 1. The circle $\alpha$ and some of the chords $A B$ for $q=4$.

Hence the set of these lines is the zero set of the function

$$
\begin{aligned}
F(t, x, y) & :=(x-\cos t)(r \sin q t-\sin t)-(y-\sin t)(r \cos q t-\cos t) \\
& =x(r \sin q t-\sin t)-y(r \cos q t-\cos t)-r \cos t \sin q t+r \sin t \cos q t \\
& =x(r \sin q t-\sin t)-y(r \cos q t-\cos t)-r \sin (q-1) t .
\end{aligned}
$$

We deduce:

$$
\frac{\partial F}{\partial t}(t, x, y)=x(r q \cos q t-\cos t)+y(r q \sin q t-\sin t)-r(q-1) \cos (q-1) t .
$$

The envelope of the straight lines $A B$ is the set of points $(x, y)$ such that there exists $t$ with $F(t, x, y)=0$ and $(\partial F / \partial t)(t, x, y)=0$ [1, Definition 5.3 p.102]. So we must solve the system

$$
\left\{\begin{array}{l}
x(r \sin q t-\sin t)-y(r \cos q t-\cos t)=r \sin (q-1) t \\
x(r q \cos q t-\cos t)+y(r q \sin q t-\sin t)=r(q-1) \cos (q-1) t .
\end{array}\right.
$$

This is a linear system in the unknown $x$ and $y$ of the form $a x-b y=u$ and $c x+d y=v$, whose solution is

$$
x=\frac{b v+d u}{a d+b c}, \quad y=\frac{a v-c u}{a d+b c}
$$

if its determinant $a d+b c$ is non zero. So we first calculate this determinant:

$$
\begin{aligned}
a d+b c= & (r \sin q t-\sin t)(r q \sin q t-\sin t)+(r \cos q t-\cos t)(r q \cos q t-\cos t) \\
= & r^{2} q \sin ^{2} q t-r \sin q t \sin t-r q \sin q t \sin t+\sin ^{2} t \\
& \quad+r^{2} q \cos ^{2} q t-r \cos q t \cos t-r q \cos q t \cos t+\cos ^{2} t \\
= & r^{2} q+1-r(q+1)(\sin q t \sin t+\cos q t \cos t) \\
= & r^{2} q+1-r(q+1) \cos (q-1) t .
\end{aligned}
$$

Suppose $a d+b c=0$; this would mean

$$
\cos (q-1) t=\frac{r^{2} q+1}{r(q+1)} .
$$

We will prove that the right-hand of (2.1) is greater than 1, which implies that (2.1) has no solution and $a d+b c \neq 0$. Let, for $r>0, f(r):=\left(r^{2} q+1\right) /(r(q+1))$. We have $f^{\prime}(r)=\left(r^{2} q-1\right) /\left(r^{2}(q+1)\right)$. Then $f^{\prime}(r)=0$ 
implies $r^{2} q-1=0$, that is, $r=1 / \sqrt{q}<1$. Since $\lim _{r \rightarrow+\infty} f^{\prime}(r)=q /(q+1)>0, f$ is increasing on $] 1 / \sqrt{q} ;+\infty[$; in particular, for all $r>1, f(r)>f(1)=1$, as wanted. Next we calculate the numerator of $x$ :

$$
b v+d u=(r \cos q t-\cos t) r(q-1) \cos (q-1) t+(r q \sin q t-\sin t) r \sin (q-1) t .
$$

We concentrate on the second term of the right hand:

$$
\begin{aligned}
(r q \sin q t-\sin t) r \sin (q-1) t= & (r q \sin q t-\sin t) r(\cos t \sin q t-\sin t \cos q t) \\
= & r^{2} q \sin ^{2} q t \cos t-r^{2} q \sin q t \sin t \cos q t \\
& -r \sin t \cos t \sin q t+r^{2} \sin ^{2} t \cos q t \\
= & r^{2} q\left(1-\cos ^{2} q t\right) \cos t-r^{2} q \sin q t \sin t \cos q t \\
& -r \sin t \cos t \sin q t+r\left(1-\cos ^{2} t\right) \cos q t \\
= & r^{2} q \cos t-r^{2} q \cos ^{2} q t \cos t-r^{2} q \sin q t \sin t \cos t \\
& \quad+r \cos q t-r \cos ^{2} t \cos q t-r \sin t \cos t \sin q t \\
= & r^{2} q \cos t-r^{2} q \cos q t(\cos q t \cos t+\sin q t \sin t) \\
& \quad+r \cos q t-r \cos t(\cos t \cos q t+\sin t \sin q t) \\
= & r^{2} q \cos t+r \cos q t-r(r q \cos q t+\cos t) \cos (q-1) t .
\end{aligned}
$$

Therefore

$$
\begin{aligned}
b v+d u= & (r \cos q t-\cos t) r(q-1) \cos (q-1) t \\
& \quad-r(r q \cos q t+\cos t) \cos (q-1) t+r^{2} q \cos t+r \cos q t \\
= & r^{2}(q-1) \cos q t \cos (q-1) t-r(q-1) \cos t \cos (q-1) t \\
& \quad-r^{2} q \cos q t \cos (q-1) t-r \cos t \cos (q-1) t+r^{2} q \cos t+r \cos q t \\
= & r^{2} q \cos t-r^{2} \cos q t \cos (q-1) t+r \cos q t-r q \cos t \cos (q-1) t \\
= & r q \cos t[r-\cos (q-1) t]+r \cos q t[1-r \cos (q-1) t] .
\end{aligned}
$$

The numerator of $y$ can be calculated along the same lines and we find:

$$
a v-c u=r q \sin t[r-\cos (q-1) t]+r \sin q t[1-r \cos (q-1) t] .
$$

We conclude that the envelope of the straight lines $A B$ is the curve $\gamma_{r}$ given by

$$
\gamma_{r}(t)=\left(\begin{array}{l}
\frac{r q \cos t[r-\cos (q-1) t]+r \cos q t[1-r \cos (q-1) t]}{r^{2} q+1-r(q+1) \cos (q-1) t} \\
\frac{r q \sin t[r-\cos (q-1) t]+r \sin q t[1-r \cos (q-1) t]}{r^{2} q+1-r(q+1) \cos (q-1) t}
\end{array}\right)
$$

for all $t \in \mathbb{R}$.

\section{Critical points.}

The critical points of $\gamma_{r}$ are those points where the derivative of $\gamma_{r}$ is zero: $\dot{\gamma}_{r}(t)=0$. Now, if the expression we have found for $\gamma_{r}$ is not simple, the one for $\dot{\gamma}_{r}$ will certainly be complicated, and to solve $\dot{\gamma}_{r}(t)=0$ from it not less.

However, it is possible to find the critical points of $\gamma_{r}$ without messy calculations if we allow ourselves the use of some old-style reasoning. Suppose $\gamma_{r}$ has a critical point at $t_{0}$. Let $\Delta t>0$ be so small that we may consider the arcs on the circles $\alpha$ and $\beta$ between $t_{-}:=t_{0}-\Delta t$ and $t_{+}:=t_{0}+\Delta t$ as straight segments. We write $A_{-}:=A\left(t_{-}\right), A_{0}:=A\left(t_{0}\right), A_{+}:=A\left(t_{+}\right)$and similarly for $B_{-}, B_{0}, B_{+}$, so that $A_{0}$ (respectively $\left.B_{0}\right)$ is the middlepoint of the segment $A_{-} A_{+}$(resp. $B_{-} B_{+}$). Let $I$ be the intersection of the straight lines $A_{-} B_{-}$and $A_{0} B_{0}$, and $J$ the intersection of $A_{0} B_{0}$ and $A_{+} B_{+}$. We have essentially two possibilities: see Figure 2 . We may consider $I$ and $J$ as points on the curve $\gamma_{r}$. The equality $\dot{\gamma}_{r}\left(t_{0}\right)=0$ means that $\gamma_{r}(t)$ does not vary for $t$ near 


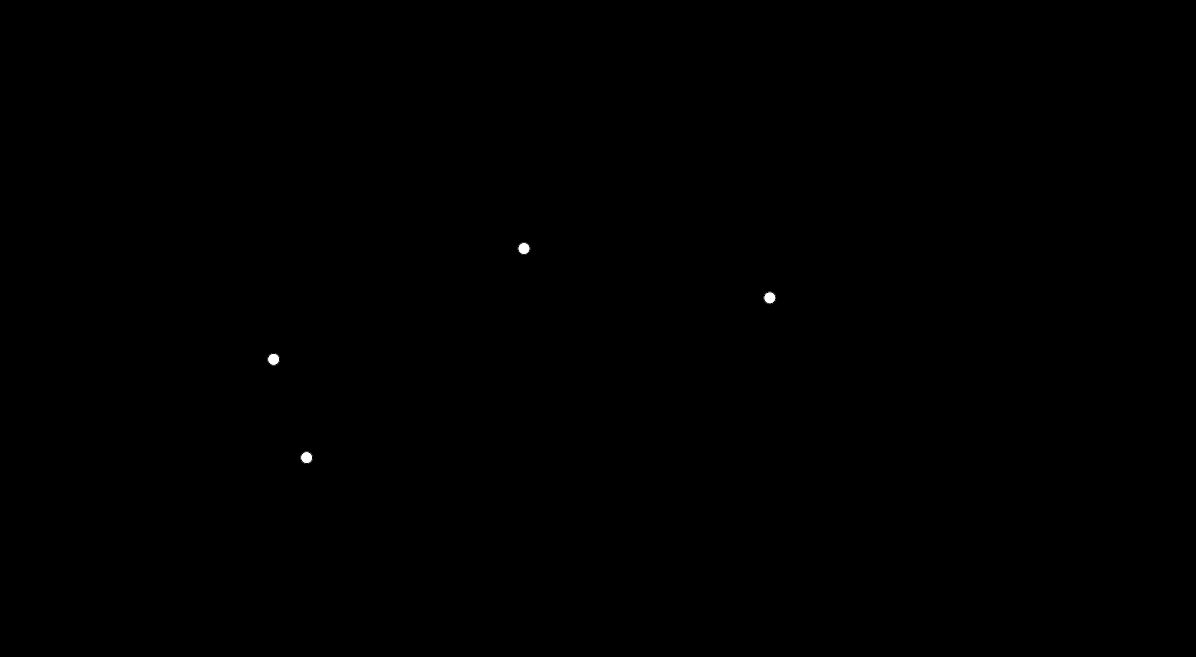

Figure 2. Position of $I$ and $J$ with respect to $A_{-} A_{+}$and $B_{-} B_{+}$

$t_{0}$; hence $I=J$, which is only possible if the segments $A_{-} A_{+}$and $B_{-} B_{+}$are parallel. Now, these segments are on the concentric circles $\alpha$ and $\beta$ respectively: the tangents at $A_{0}$ to $\alpha$ and at $B_{0}$ to $\beta$ can only be parallel when $A_{0}$ and $B_{0}$ are aligned with the centre $O$. Going back to the parametrizations of $A$ and $B$, this gives two possibilities:

(a) $\cos q t_{0}=-\cos t_{0}$ and $\sin q t_{0}=-\sin t_{0}$ ；

(b) $\cos q t_{0}=\cos t_{0}$ and $\sin q t_{0}=\sin t_{0}$.

The case (a) occurs when $q t_{0}=t_{0}+(2 l+1) \pi$ for some $l \in \mathbb{Z}$, that is,

$$
t_{0}=\frac{(2 l+1) \pi}{q-1} \text {. }
$$

Then $\cos (q-1) t_{0}=\cos (2 l+1) \pi=-1$ and

$$
x=\frac{r q \cos t_{0}[r+1]-r \cos t_{0}[1+r]}{r^{2} q+1+r(q+1)}=\frac{r(r+1)(q-1) \cos t_{0}}{(r q+1)(r+1)}=\frac{r(q-1) \cos t_{0}}{r q+1} .
$$

Similarly,

$$
y=\frac{r(q-1) \sin t_{0}}{r q+1} .
$$

Hence all these critical points are on the circle with centre $O$ and radius

$$
R_{a}:=\frac{r(q-1)}{r q+1} .
$$

The case (b) occurs when $q t_{0}=t_{0}+2 l \pi$ for some $l \in \mathbb{Z}$, that is,

$$
t_{0}=\frac{2 l \pi}{q-1} .
$$

Then $\cos (q-1) t_{0}=\cos 2 l \pi=1$ and

$$
x=\frac{r q \cos t_{0}[r-1]+r \cos t_{0}[1-r]}{r^{2} q+1-r(q+1)}=\frac{r(r-1)(q-1) \cos t_{0}}{(r q-1)(r-1)}=\frac{r(q-1) \cos t_{0}}{r q-1} .
$$

Similarly,

$$
y=\frac{r(q-1) \sin t_{0}}{r q-1} .
$$


Hence all these critical points are on the circle with centre $O$ and radius

$$
R_{b}:=\frac{r(q-1)}{r q-1} .
$$

Moreover, $R_{a}<R_{b}<1$.

\section{Periodicity.}

Suppose $q$ is a rational number: $q=m / n$ with $m, n \in \mathbb{Z}, m>n \geq 1$ and $\operatorname{gcd}(m, n)=1$. We show that $\gamma_{r}$ is in this case periodic and therefore its image is a closed curve.

To find the period of $\gamma_{r}$, we must find when the points $A$ and $B$ have regained their initial position at $(1,0)$ and $(r, 0)$ respectively, that is, when both points have toured a whole number of times around $O$. As $A$ tours $n$ times, $B$ tours $q \cdot n=\frac{m}{n} \cdot n=m$ times: they are at their initial position. Moreover, since $\operatorname{gcd}(m, n)=1$, this cannot occur before. Hence $\gamma_{r}$ is periodic of period $2 n \pi$. (This does not preclude the curve intersecting itself for $t$ strictly between 0 and $2 n \pi$; in fact, when $n>1$ some selfintersection is necessary.) The critical points of type (a) (see section 3) occur at

$$
t=\frac{(2 l+1) \pi}{m / n-1}=\frac{(2 l+1) n \pi}{m-n} .
$$

It follows that there are $m-n$ distinct critical points of type (a), corresponding to $l=0, \ldots, m-n-1$. The critical points of type (b) (see section 3) occur at

$$
t=\frac{2 l \pi}{m / n-1}=\frac{2 \ln \pi}{m-n} .
$$

It follows that there are $m-n$ distinct critical points of type (b), corresponding to $l=0, \ldots, m-n-1$. In total, $\gamma_{r}$ has $2(m-n)$ critical points.

When $q$ is not rational, $\gamma_{r}$ is not periodic, so its image is not a closed curve, and it has infinitely many critical points of both types.

\section{Within the unit circle.}

First, we prove that $\gamma_{r}$ cannot go outside the circle $\beta$. Let $t_{0}$ be such that $\gamma_{r}\left(t_{0}\right)$ is one of the points on the curve which are farthest from $O$, and suppose $\gamma_{r}\left(t_{0}\right)$ is outside $\beta$. Then $t_{0}$ cannot be a critical point, since all critical points are inside $\alpha$ (see section 3). Hence it is regular and $\dot{\gamma}_{r}\left(t_{0}\right) \neq 0$, so the tangent to the curve $\gamma_{r}$ at $\gamma_{r}\left(t_{0}\right)$ is well defined. Moreover, $\dot{\gamma}_{r}\left(t_{0}\right)$ is orthogonal to $\gamma_{r}\left(t_{0}\right)$ : since $\left\|\gamma_{r}(t)\right\|^{2}$ is maximal at $t_{0}$, its derivative at $t_{0}$ is zero, i.e. $2 \gamma_{r}\left(t_{0}\right) \dot{\gamma}_{r}\left(t_{0}\right)=0$. But the curve $\gamma_{r}$ is tangent at $\gamma_{r}\left(t_{0}\right)$ to the line given by $F\left(t_{0}, x, y\right)=0$ [1, proposition 5.25 p.114]. Now it follows from the first part of our discussion that the tangent to the curve at $\gamma_{r}\left(t_{0}\right)$ does not touch the circle $\beta$, and hence cannot be a straight line through $A$ and $B$ : contradiction.

The same argument shows that $\gamma_{r}$ cannot go outside the circle $\alpha$ : see Figure 3.

Next we show that there are points of $\gamma_{r}$ which lie on $\alpha$. We already know that, if such a point $\gamma_{r}(t)$ exists, $\dot{\gamma}_{r}(t) \neq 0$ and $\gamma_{r}(t)$ it is farthest from the centre $O$ and hence $\dot{\gamma}_{r}(t)$ is orthogonal to $\gamma_{r}(t)$. Moreover the tangent to $\gamma_{r}$ at $\gamma_{r}(t)$ is the straight line given by $F(t, x, y)=0$, in other words: it goes through $A(t)$ and $B(t)$. This implies $\gamma_{r}(t)=A(t)$, i.e.

$$
\left\{\begin{array}{l}
\frac{r q \cos t[r-\cos (q-1) t]+r \cos q t[1-r \cos (q-1) t]}{r^{2} q+1-r(q+1) \cos (q-1) t}=\cos t \\
\frac{r q \sin t[r-\cos (q-1) t]+r \sin q t[1-r \cos (q-1) t]}{r^{2} q+1-r(q+1) \cos (q-1) t}=\sin t
\end{array}\right.
$$

The first equation can be written:

$$
r^{2} q \cos t-r q \cos t \cos (q-1) t+r \cos q t[1-r \cos (q-1) t]=r^{2} q \cos t+\cos t-r(q+1) \cos t \cos (q-1) t
$$

that is,

$$
r \cos q t[1-r \cos (q-1) t]-\cos t+r \cos t \cos (q-1) t=0
$$




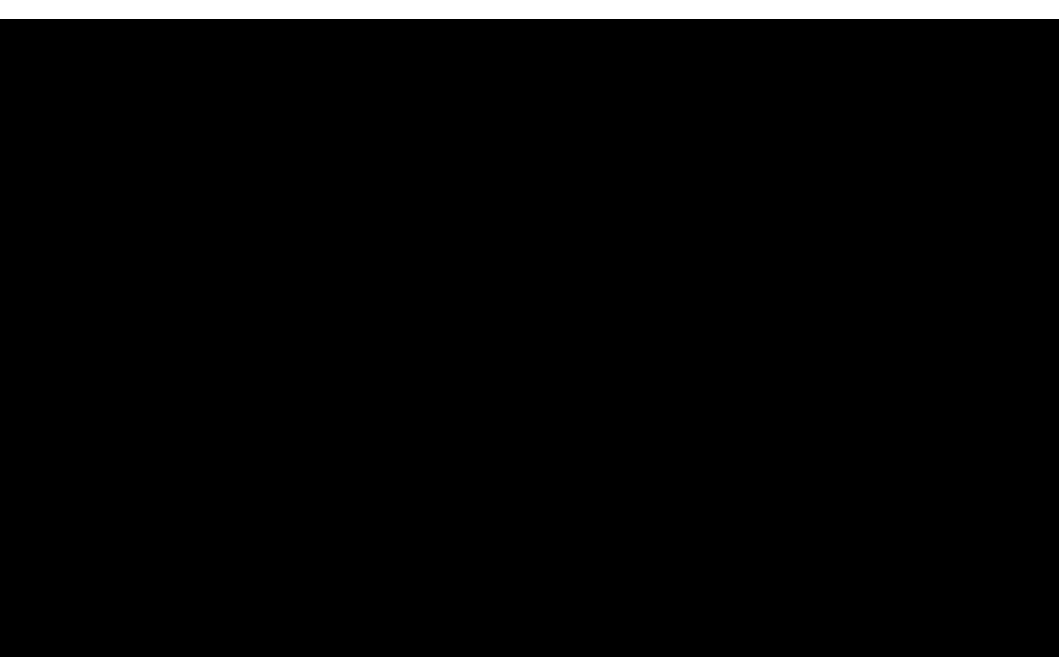

Figure 3. The curve $\gamma_{r}$ cannot go outside $\alpha$.

or

$$
[r \cos q t-\cos t] \cdot[1-r \cos (q-1) t]=0 .
$$

The calculations with the second equation of (5.1) are analogue and we get the system

$$
\left\{\begin{array}{l}
{[r \cos q t-\cos t] \cdot[1-r \cos (q-1) t]=0} \\
{[r \sin q t-\sin t] \cdot[1-r \cos (q-1) t]=0 .}
\end{array}\right.
$$

If $1-r \cos (q-1) t \neq 0$, we have

$$
\cos q t=\frac{1}{r} \cos t, \quad \sin q t=\frac{1}{r} \sin t
$$

and

$$
\cos (q-1) t=\cos q t \cos t+\sin q t \sin t=\frac{1}{r} \cos ^{2} t+\frac{1}{r} \sin ^{2} t=\frac{1}{r} .
$$

We conclude that the curve $\gamma_{r}$ touches (and is tangent to) the circle $\alpha$ exactly for every $t$ with $\cos (q-1) t=1 / r$.

(When $\gamma_{r}$ is periodic, the existence of points on the curve for which $\left\|\gamma_{r}(t)\right\|^{2}$ is maximal is clear by compactness. In the aperiodic case, one must reason on a piece of the curve joining two critical points.)

\section{Away from the origin.}

We prove here that there is no point on the curve whose distance from $O$ is less than $R_{a}$. Suppose such a point exists: we may even choose $t$ such that $\left\|\gamma_{r}(t)\right\|^{2}$ be minimal. Then $t$ cannot be a critical point (see section 3). Hence $\dot{\gamma}_{r}(t) \neq 0$, so the tangent to the curve $\gamma_{r}$ at $\gamma_{r}(t)$ is well defined. Moreover, $\dot{\gamma}_{r}(t)$ is orthogonal to $\gamma_{r}(t)$ and the tangent to the curve at $\gamma_{r}(t)$ is the straight line through $A(t)$ and $B(t)$, by a reasoning already used in section 5. This implies that the scalar product of $\gamma_{r}(t)$ with the vector joining $A(t)$ to $B(t)$ is zero:

$$
0=\left(\begin{array}{l}
\frac{r q \cos t[r-\cos (q-1) t]+r \cos q t[1-r \cos (q-1) t]}{r^{2} q+1-r(q+1) \cos (q-1) t} \\
\frac{r q \sin t[r-\cos (q-1) t]+r \sin q t[1-r \cos (q-1) t]}{r^{2} q+1-r(q+1) \cos (q-1) t}
\end{array}\right) \cdot\left(\begin{array}{l}
r \cos q t-\cos t \\
r \sin q t-\sin t
\end{array}\right) .
$$

Multiplying by the common denominator (which is never zero), we have

$$
\begin{aligned}
0= & r^{2} q \cos t \cos q t[r-\cos (q-1) t]-r q \cos ^{2} t[r-\cos (q-1) t] \\
& +r^{2} \cos ^{2} q t[1-r \cos (q-1) t]-r \cos q t \cos t[1-r \cos (q-1) t] \\
+ & r^{2} q \sin t \sin q t[r-\cos (q-1) t]-r q \sin ^{2} t[r-\cos (q-1) t] \\
& +r^{2} \sin ^{2} q t[1-r \cos (q-1) t]-r \sin q t \sin t[1-r \cos (q-1) t],
\end{aligned}
$$


Or

$$
\begin{aligned}
0= & \left\{r^{2} q \cos t \cos q t+r^{2} q \sin t \sin q t-r q \cos ^{2} t-r q \sin ^{2} t\right\}[r-\cos (q-1) t] \\
& +\left\{r^{2} \cos ^{2} q t+r^{2} \sin ^{2} q t-r \cos q t \cos t-r \sin q t \sin t\right\}[1-r \cos (q-1) t] .
\end{aligned}
$$

We simplify it to

$$
0=\left\{r^{2} q \cos (q-1) t-r q\right\}[r-\cos (q-1) t]+\left\{r^{2}-r \cos (q-1) t\right\}[1-r \cos (q-1) t],
$$

that is,

$$
0=r q\{r \cos (q-1) t-1\}[r-\cos (q-1) t]+r\{r-\cos (q-1) t\}[1-r \cos (q-1) t],
$$

or

$$
0=(r q-r)[r-\cos (q-1) t] \cdot[r \cos (q-1) t-1],
$$

But $r-\cos (q-1) t=0$ has no solution since $r>1$; and the solutions to $r \cos (q-1) t-1=0$ give the points on $\alpha$ already found. The conclusion follows.

\section{The limit case $r=1$.}

When $r$ tends to 1 , the expression (2.2) for $\gamma_{r}$ gives

$$
\gamma_{1}(t)=\left(\begin{array}{l}
\frac{q \cos t[1-\cos (q-1) t]+\cos q t[1-\cos (q-1) t]}{q+1-(q+1) \cos (q-1) t} \\
\frac{q \sin t[1-\cos (q-1) t]+\sin q t[1-\cos (q-1) t]}{q+1-(q+1) \cos (q-1) t}
\end{array}\right) .
$$

The terms $1-\cos (q-1) t$ (which give a possible zero of the denominator) cancel out to

$$
\gamma_{1}(t)=\left(\begin{array}{c}
\frac{q \cos t+\cos q t}{q+1} \\
\frac{q \sin t+\sin q t}{q+1}
\end{array}\right) .
$$

Comparing with the representation of an epicycloid in [2, volume II, formula (8) p.162], we see that $\gamma_{1}$ is the epicycloid obtained as the trajectory of a point on a circle of radius $\rho:=1 /(q+1)$ rolling around a circle of radius $R:=(q-1) /(q+1)$. Note that $R+2 \rho=1$ and that the ratio $R / \rho$ equals $q-1$. We observe further (see (3.1) and (3.2)) that

$$
\lim _{r \rightarrow 1_{+}} R_{a}=R, \quad \lim _{r \rightarrow 1_{+}} R_{b}=1 .
$$

This means that the critical points of type $(a)$ remain but that those of type $(b)$ disappear: they merge with the points of contact of $\gamma_{r}$ with $\alpha$ in those particular points where $\cos t_{0}=\cos q t_{0}$ and $\sin t_{0}=\sin q t_{0}$, i.e. $A\left(t_{0}\right)=B\left(t_{0}\right)$ where there is no chord possible! In fact, the envelope is not defined at these points: we closed the gap by continuity (these points correspond to the zeros of the term $1-\cos (q-1) t$ in our first expression for $\left.\gamma_{1}\right)$.

\section{The limit case $r=+\infty$.}

When $r$ tends to $+\infty$, the expression (2.2) for $\gamma_{r}$ gives

$$
\gamma_{\infty}(t)=\left(\begin{array}{l}
\frac{q \cos t-\cos q t \cos (q-1) t}{q} \\
\frac{q \sin t-\sin q t \cos (q-1) t}{q}
\end{array}\right) .
$$




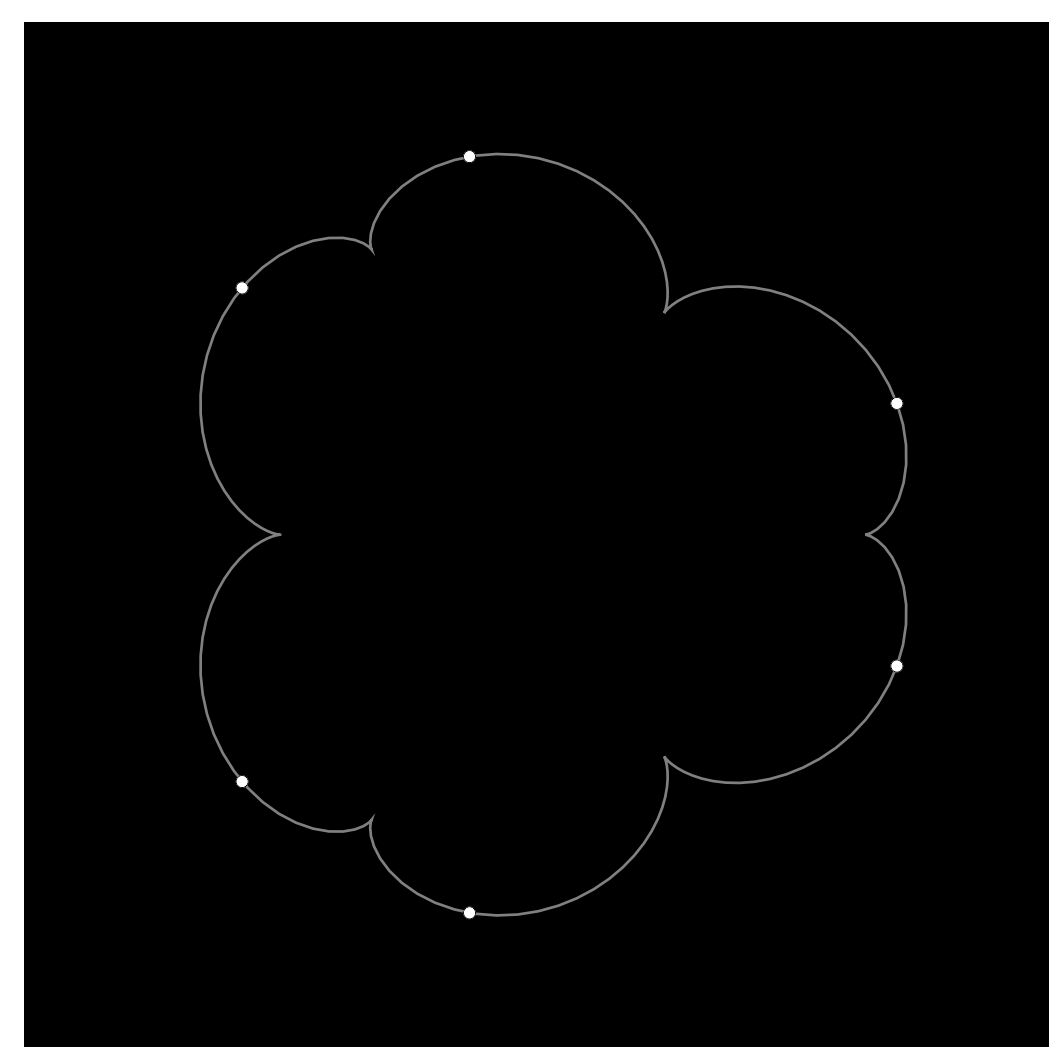

Figure 4. The case $q=4$ and $r=2$ in detail.

Now

$$
\cos q t \cos (q-1) t=\frac{1}{2} \cos (2 q-1) t+\frac{1}{2} \cos t
$$

hence

$$
q \cos t-\cos q t \cos (q-1) t=\frac{1}{2}[(2 q-1) \cos t-\cos (2 q-1) t] .
$$

An analogue calculation for the second coordinate gives finally

$$
\gamma_{\infty}(t)=\left(\begin{array}{l}
\frac{(2 q-1) \cos t-\cos (2 q-1) t}{2 q} \\
\frac{(2 q-1) \sin t-\sin (2 q-1) t}{2 q}
\end{array}\right) .
$$

Comparing with the representation of an epicycloid in [2, volume II, formula (2) p.156], we see that $\gamma_{\infty}$ is the epicycloid obtained as the trajectory of a point on a circle of radius $\rho:=1 / 2 q$ rolling around a circle of radius $R:=(q-1) / q$. Note that $R+2 \rho=1$ and that the ratio $R / \rho$ equals $2(q-1)$, which is the double of the ratio $R / \rho$ in the case $r=1$. We observe further (see (3.1) and (3.2)) that

$$
\lim _{r \rightarrow+\infty} R_{a}=R, \quad \lim _{r \rightarrow+\infty} R_{b}=R .
$$

This means that all critical points of $\gamma_{r}$ remain, but the distinction between type $(a)$ and type $(b)$ disappears.

That in this case we also have an epiycloid is not a surprise. When $r$ tends to $+\infty$, the straight line $A B$ tends to a line through $A$ which makes an angle $q t$ with the $x$-axis. We can then use [2, volume II, §560 p.165] or [3, p.140]: the diameter of a circle rolling around another circle envelopes an epicycloid. 

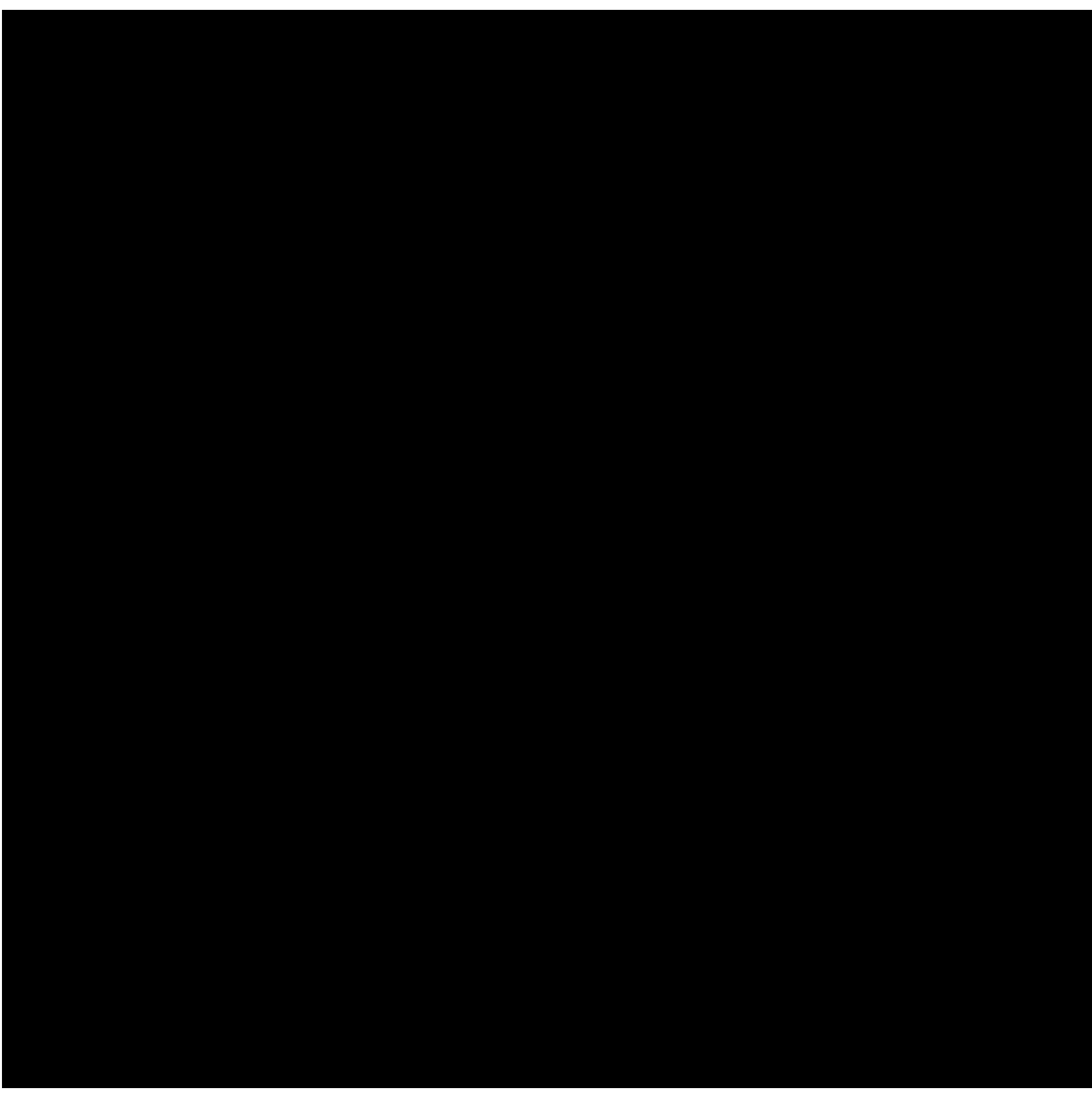

Figure 5. With $q=4$, top left: $r=7 / 6$, top right: $r=5 / 3$, bottom left: $r=3$, bottom right: $r=30$.

\section{Examples.}

We take $q=4$ and $r=2$. The curve $\gamma_{2}$ has period $2 \pi$. The three critical points of type (a) are on the circle of radius $R_{a}=2 / 3$ at angles $\pi / 3, \pi$ and $5 \pi / 3$. The three critical points of type (b) are on the circle of radius $R_{b}=6 / 7$ at angles $0,2 \pi / 3$ and $4 \pi / 3$. The points of contact of $\gamma_{2}$ with $\alpha$ are at the angles $t$ such that $\cos 3 t=1 / 2$ i.e. $\cos 3 t=\cos \pi / 3$; we find the six angles $\pi / 9,5 \pi / 9,7 \pi / 9,11 \pi / 9,13 \pi / 9$ and $17 \pi / 9$. All this can be seen in Figure 4.

In Figure 5 we illustrate, also for $q=4$, the cases $r=7 / 6, r=5 / 3, r=3$ and $r=30$, without drawing the curve $\gamma_{r}$ itself.

Sections 7 and 8 and the preceding example may suggest that the epicycloid for $r=+\infty$ shows two times more critical points than the one for $r=1$. It is not always so. Take $q=3 / 2$; for $r=1$ as well as for $r=+\infty$ the epicycloids show one critical point. 


\section{Another natural morphing.}

Almost all calculations made in the preceding sections for $q>1$ are still valid when $q<-1$, that is, when $B$, starting from $(r, 0)$, moves on $\beta$ in the sense opposite to $A$. We only state some salient differences in the results: $1<R_{b}<R_{a}$; if $q=-m / n$ with $m, n \in \mathbb{Z}, m>n \geq 1$ and $\operatorname{gcd}(m, n)=1, \gamma_{r}$ has $m+n$ critical points of type (a) and $m+n$ critical points of type (b); the curve $\gamma_{r}$ is again tangent to the circle $\alpha$ but does not go inside it; finally, the limit cases $\gamma_{1}$ and $\gamma_{\infty}$ are hypocycloids.

\section{References}

[1] Bruce, J. W. and Giblin, P. J., Curves and singularities (2nd edition). Cambridge University Press, Cambridge, 1992.

[2] Gomes Teixeira, F., Traité des courbes spéciales remarquables planes et gauches (reprint). Chelsea Publishing Company, New York, 1971.

[3] Lockwood, E. H., A book of curves. Cambridge University Press, Cambridge, 1961.

[4] Sewell, M., Parametric Envelopes. Math. Gazette 99 (2015), 226-229.

\section{Affiliations}

F. J. GONZÁLEZ VIELI

ADDRESS: Montoie 45, 1007 Lausanne, Switzerland

E-MAIL: francisco-javier.gonzalez@gmx.ch

ORCID ID : orcid.org/0000-0002-2245-5757 